\title{
Interval Estimations of Global Weights in AHP by Upper Approximation
}

\author{
Tomoe Entani ${ }^{a}$ Hideo Tanaka ${ }^{\mathrm{b}}$ \\ a Kochi University \\ 2-5-1 Akebono Kochi 780-8520, JAPAN \\ TEL: +81-88-844-8212, FAX: +81-88-844-8225 \\ Email:entani@cc.kochi-u.ac.jp \\ b Hiroshima International University \\ Gakuendai 555-36,Kurose, Hiroshima 724-0695, JAPAN \\ TEL: +81-823-70-4886, FAX: +81-823-70-4852 \\ Email: h-tanaka@he.hirokoku-u.ac.jp
}

\begin{abstract}
In Analytic Hierarchy Process (AHP) structured hierarchically as several criteria and alternatives, the priority of an alternative is obtained by using the pairwise comparisons based on decision maker's intuition. Thus, the given comparisons are uncertain and inconsistent each other. We use the interval approach for obtaining interval evaluations which are suitable for handling uncertain data. Since the given comparisons are ratio measures and too large intervals are not useful information, the intervals should be normalized and their redundancy should be reduced. We introduce interval probability which fills the role of interval normalization instead of crisp normalization in the estimations at each hierarchy. Then, as a final decision, the interval global weights reflecting a decision maker's uncertain judgements as their widths without redundancy are obtained.
\end{abstract}

Key words: Analytic hierarchy process, Interval local and global weights, Interval referenced priority weights, Upper approximations

\section{Introduction}

Analytic Hierarchy Process (AHP) is a useful method in multi-criteria decision making problems [4]. It is structured hierarchically as criteria and alternatives and proposed to determine the priority weights of alternatives which are called the global weights. From a pairwise comparison matrix for criteria, the referenced priority weights are obtained by the eigenvector method [4]. In the 
same way, from a pairwise comparison matrix for alternatives under each criterion, the local weights for the criterion are obtained. The elements of the two types of comparison matrices are relative measurements given by a decision maker. The obtained weights from the matrix can reflect his/her attitude in the actual decision problem. The weights obtained by the conventional AHP lead to a linear order of alternatives. Uncertainty of an order of alternatives in AHP is discussed in [5]. As shown in [5], there exists a problem that pairwise comparisons might be uncertain and inconsistent with each other because they are based on human intuition. The approaches for dealing with interval and fuzzy comparisons have been proposed in [1] and [3], respectively. It is easier for a decision maker to give interval or fuzzy comparisons than crisp ones, since they are suitable to represent uncertain human judgements. The approach with interval weights [1] is rather complex comparing to our approach which can handle interval data, because the approach [1] is based on solving problems on all vertices for obtaining interval weights. In case of fuzzy comparisons [3], the optimal weight pattern is obtained as crisp by maximizing the degree of consistency in a sense of fuzzy comparisons. Although we propose the model to obtain interval weights from the crisp comparisons, the model can be extended to interval comparisons and the obtained weights are intervals. In the similar setting to [1], the approaches for dealing with decision maker's preference statements instead of pairwise comparisons are proposed in [6]. This seems to be very practical, but obtaining the upper and lower bounds of interval weights has been proposed without defining the interval weights.

In this paper, it is assumed that the estimated referenced priority and local weights are intervals to reflect inconsistency of the given pairwise comparisons. The widths of the obtained interval weights can be regarded as the index of inconsistency among the given comparisons. If the consistent comparisons are given, we can obtain crisp weights by the proposed approach and they are the same as ones obtained by eigenvector method [7]. With the obtained interval referenced priority and local weights, the global weights of all alternatives are also obtained as intervals. However, their widths tend to be large if we use interval arithmetic. Thus, the obtained intervals by interval arithmetic can not be a meaningful as long as unnecessary widths of intervals are not removed. The optimization problems to obtain global (aggregated) evaluation as fuzzy number is proposed in [3], where the utilities under criteria representing the local weighs are crisp and the optimal weight pattern representing the referenced priority weights is determined from the given fuzzy comparisons. We propose to obtain the global weights of alternatives as follows: first the crisp referenced priority weight within the obtained interval one is multiplied by the interval local weight under the criterion and then they are added over all criteria. The crisp referenced priority weights are selected such that their sum becomes one. The interval global weights are also obtained as interval probabilities so that they are normalized to reduce redundancy. The normalized interval global weights are useful information for a final decision in the sense 
that they reflect a decision maker's uncertain judgements as intervals without redundancy.

\section{Crisp Weights by Conventional Eigenvector Method}

AHP is a method to deal with the weights with respect to many alternatives and to determine the priority weight of each alternative [4]. When there are $n$ alternatives, a decision maker compares a pair of alternatives for all possible pairs to obtain a pairwise comparison matrix $A$ as follows.

$$
A=\left[a_{i j}\right]=\left(\begin{array}{ccc}
1 & \cdots & a_{1 n} \\
\vdots & a_{i j} & \vdots \\
a_{n 1} & \cdots & 1
\end{array}\right)
$$

where $a_{i j}$ shows the priority ratio of alternative $i$ comparing to alternative $j$ and they satisfy the following relations so that the decision maker gives $n(n-1) / 2$ comparisons.

$$
\begin{aligned}
& \text { Diagonal elements } \quad a_{i i}=1 \\
& \text { Reciprocal elements } a_{i j}=1 / a_{j i}
\end{aligned}
$$

where it follows from the reciprocal relation that the assumed model is a ratio model.

From the given comparison matrix, the crisp priority weights $w_{i}^{*}(i=1, \ldots, n)$ are obtained by eigenvector method. The eigenvector problem is formulated as follows.

$$
A \boldsymbol{w}=\lambda \boldsymbol{w}
$$

where $\lambda$ is the eigenvalue and $\boldsymbol{w}$ is the eigenvector. Solving (2), the eigenvector $\boldsymbol{w}^{*}=\left(w_{1}^{*}, \ldots, w_{n}^{*}\right)^{t}$ corresponding to the principal eigenvalue is obtained as the priority weight vector. It is noted that the sum of the obtained weights $w_{i}^{*}(i=1, \ldots, n)$ is normalized to be one. 


\section{Interval Weights by Approximation Models}

The given pairwise comparison $a_{i j}$ is approximated by the ratio of priority weights, $w_{i}$ and $w_{j}$, symbolically written as $a_{i j} \approx w_{i} / w_{j}$.

Assuming the priority weight $w_{i}$ as an interval, we obtain the interval priority weights denoted as $W_{i}=\left[\underline{w}_{i}, \bar{w}_{i}\right]$. Then, the approximated pairwise comparison with the interval weights is defined as the following interval.

$$
\frac{W_{i}}{W_{j}}=\left[\underline{\underline{w}}_{i}, \frac{\bar{w}_{i}}{\underline{w}_{j}}\right]
$$

where the upper and lower bounds of the approximated comparison are defined as the maximum range.

\subsection{Interval Normalization}

First it should be noted that the sum of weights obtained by the conventional AHP is normalized to be one. Therefore, we consider interval probability proposed in [9] so as to normalize the interval weights. Their conditions are defined as follows.

Definition 1 Interval weights $\left(W_{1}, \ldots, W_{n}\right)$ are called interval probabilities if and only if

$$
\begin{aligned}
& \sum_{i \neq j} \bar{w}_{i}+\underline{w}_{j} \geq 1 \quad \forall j \\
& \sum_{i \neq j} \underline{w}_{i}+\bar{w}_{j} \leq 1 \quad \forall j
\end{aligned}
$$

where $W_{i}=\left[\underline{w}_{i}, \bar{w}_{i}\right]$.

It can be said that the conventional normalization which makes the sum of values is one is extended to the interval normalization by using the above conditions. The sums of the bounds of intervals are constrained by one in two ways. These conditions make the intervals meaningful in the sense that there are elements in the intervals so as to make the sum of them be one. In order to make intervals be normalized, their redundancy should be reduced by (3) as the constraint conditions in the optimization problem described in the subsection 3.2 . 


\subsection{Upper Approximations of Crisp Pairwise Comparisons}

The proposed approach is based on the view that the interval weights are obtained so as to include the given interval [7]. The obtained interval weights satisfy the following inclusion relations.

$$
a_{i j} \subseteq \frac{W_{i}}{W_{j}}=\left[\frac{\underline{w}_{i}}{\bar{w}_{j}}, \frac{\bar{w}_{i}}{\underline{w}_{j}}\right] \quad \forall(i, j)
$$

which means

$$
\frac{\underline{\underline{w}}_{i}}{\bar{w}_{j}} \leq a_{i j} \leq \frac{\bar{w}_{i}}{\underline{w}_{j}} \forall(i, j)
$$

The approximated interval weights should be estimated as closely as possible to the given comparisons subject to the above inclusion relations. The concept of the least upper approximation [8], that is the width of each interval weight must be minimized, is applied. In the following LP problem, simply the sum of widths of all weights is minimized under the constraints.

$$
\begin{array}{cl} 
& \min \quad \sum_{i}\left(\bar{w}_{i}-\underline{w}_{i}\right) \\
\text { s.t. } & \sum_{i \neq j} \bar{w}_{i}+\underline{w}_{j} \geq 1 \quad \forall j \\
& \sum_{i \neq j} \underline{w}_{i}+\bar{w}_{j} \leq 1 \quad \forall j \\
\underline{\underline{w}}_{i} \leq a_{i j} \leq \frac{\bar{w}_{i}}{\underline{w}_{j}} \forall(i, j) \\
\bar{w}_{j} \\
\bar{w}_{i} \geq \underline{w}_{i} \geq \epsilon \quad \forall i
\end{array}
$$

where constraint conditions consist of the interval normalization (3) and inclusion relations (4). (5) is called the upper approximation model.

The interval weights by (5) include the given inconsistent comparisons data. The width of the interval represents uncertainty of each weight and the least uncertain weights are obtained by solving (5).

\subsection{Interval Global Weights}

The decision problem in AHP is structured hierarchically as criteria and alternatives as in Fig.1.

A decision maker gives pairwise comparison matrix for alternatives $\mathrm{A}_{i}(i=$ 


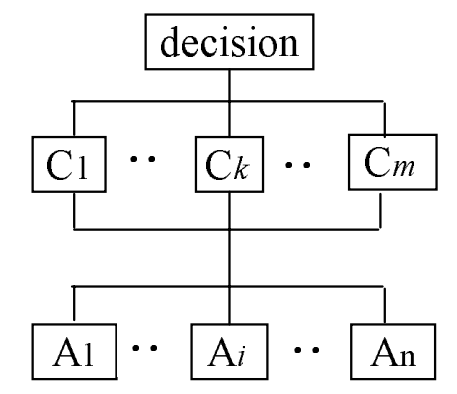

Fig. 1. Structure of decision problem in AHP

$1, \ldots, n)$ under each criterion and also comparison matrix for criteria $\mathrm{C}_{k}(k=$ $1, \ldots, m)$ comparing alternatives and criteria importance, respectively. In Fig.1, the criteria are at one layer, however, it is possible to construct several layers of criteria. By the proposed approximation model, the local weight of alternative $\mathrm{A}_{i}$ under criterion $\mathrm{C}_{k}$ is denoted as $W_{k i}=\left[\underline{w}_{k i}, \bar{w}_{k i}\right]$ and the referenced priority weight of criterion $\mathrm{C}_{k}$ is denoted as $P_{k}=\left[\underline{p}_{k}, \bar{p}_{k}\right]$. The global weight of alternative $\mathrm{A}_{i}$ is obtained as $W_{i}=\sum_{k} P_{k} W_{k i}$ by interval arithmetic. It is the sum of multiplications of the referenced priority weights and corresponding local weights so that it represents the priority of an alternative considering all criteria. In case of several layers of criteria, the global weights can be obtained by repeating the similar calculation. The local and referenced priority weights are intervals so that the global weights tend to be obtained as large intervals because of interval arithmetic. Therefore, we consider that the global weight is obtained by the crisp referenced priority weights, whose sum is one, within the interval weights. The upper bound of interval global weight of the alternative $\mathrm{A}_{i}$ is obtained as follows.

$$
\begin{aligned}
\bar{w}_{i}= & \max \sum_{k} p_{k}^{i *} \bar{w}_{k i} \\
\text { s.t. } & \sum_{k} p_{k}^{i *}=1 \\
& \underline{p}_{k} \leq p_{k}^{i *} \leq \bar{p}_{k} \quad \forall k
\end{aligned}
$$

where $p_{k}^{i *}(k=1, \ldots, m)$ are decision variables for the crisp referenced priority weights of criteria that maximize the upper bound of the interval global weight of the alternative $\mathrm{A}_{i}$.

Similarly, the problem to obtain the lower bound of interval global weight of 
the alternative $A_{i}$ is formulated as follows.

$$
\begin{aligned}
\underline{w}_{i}= & \min \sum_{k} p_{k *}^{i} \underline{w}_{k i} \\
\text { s.t. } & \sum_{k} p_{k *}^{i}=1 \\
& \underline{p}_{k} \leq p_{k *}^{i} \leq \bar{p}_{k} \quad \forall k
\end{aligned}
$$

The interval global weight of the alternative $\mathrm{A}_{i}$ can be denoted as $W_{i}=$ $\left[\underline{w}_{i}, \bar{w}_{i}\right]$. The bounds satisfy $p_{k}^{i *} \bar{w}_{k i} \leq p_{k *}^{i} \underline{w}_{k i}$ because of maximizing and minimizing the objective functions, respectively. It represents the possible range under the condition that the sum of referenced priority weights is one.

The interval global weights reflect inconsistency in the given comparisons for criteria and alternatives under each criterion. The crisp referenced priority weights are determined by maximizing and minimizing the global weight of each alternative $\mathrm{A}_{i}$, respectively. The selected crisp weights for the upper and lower bounds of interval global weight are different each other. (6) and (7) are formulated for each alternative $\mathrm{A}_{i}$ so that the crisp referenced priority weights are also different among alternatives. They depend on local weights of the alternative under the criteria. The obtained interval global weights satisfy the conditions in (3). This fact is proved in Appendix. By the proposed models for global weights (6) and (7), the interval global weights are obtained as interval probabilities, that is, they are normalized.

\section{Numerical Example}

There are 6 alternatives (A1, A2, A3, A4, A5, A6) and 6 criteria (C1, C2, C3, C4, C5, C6). A decision maker gives pairwise comparison matrices for 6 criteria and 6 alternatives under each criterion. Then, the global weights of all alternatives are calculated from them by the proposed models.

The decision maker compares all the pairs of criteria and gives the comparisons. The pairwise comparison matrix for 6 criteria followed by (1) is as 
follows.

$$
\text { Pairwise comparison matrix for criteria }=\left(\begin{array}{cccccc}
1 & 1 / 9 & 1 / 8 & 1 / 9 & 1 / 9 & 1 / 9 \\
9 & 1 & 2 & 1 / 5 & 1 / 6 & 6 \\
8 & 1 / 2 & 1 & 1 / 7 & 1 / 6 & 5 \\
9 & 5 & 7 & 1 & 2 & 7 \\
9 & 6 & 6 & 1 / 2 & 1 & 5 \\
9 & 1 / 6 & 1 / 5 & 1 / 7 & 1 / 5 & 1
\end{array}\right)
$$

The interval referenced priority weights obtained from the above matrix by the upper approximation model (5) are shown in Table 1.

Table 1

Interval referenced priority weights of criteria

\begin{tabular}{ccc}
\hline Criterion & Referenced priority & Width \\
\hline C1 & {$[0.008,0.056]$} & 0.048 \\
C2 & {$[0.042,0.101]$} & 0.059 \\
C3 & {$[0.042,0.072]$} & 0.030 \\
C4 & 0.505 & - \\
C5 & 0.253 & - \\
C6 & {$[0.014,0.072]$} & 0.058 \\
\hline
\end{tabular}

The decision maker also gives 6 pairwise comparison matrices for alternatives. As an example, the pairwise comparison matrix under $\mathrm{C} 1$ followed by (1) is as follows.

Pairwise comparison matrix for alternatives under the criterion $\mathrm{C} 1$

$$
=\left(\begin{array}{cccccc}
1 & 1 & 1 / 6 & 2 & 1 & 1 / 7 \\
1 & 1 & 1 / 6 & 2 & 1 & 1 / 7 \\
6 & 6 & 1 & 7 & 6 & 1 / 2 \\
1 / 2 & 1 / 2 & 1 / 7 & 1 & 1 / 2 & 1 / 8 \\
7 & 1 & 1 / 6 & 2 & 1 & 1 / 8 \\
7 & 7 & 2 & 8 & 8 & 1
\end{array}\right)
$$

The interval local weights obtained from the above matrix is $\boldsymbol{W}_{1}=([0.042,0.072],[0.042,0.072], 0.253,[0.032,0.063],[0.042,0.063], 0.507)^{t}$. 
In the same way, the decision maker gives pairwise comparison matrices under other criteria and the obtained interval local weights are shown in Table 2.

Table 2

Interval local weights of alternatives under each criterion

Local weight

\begin{tabular}{cccc} 
Alternative & C1 & C2 & C3 \\
\hline A1 & {$[0.042,0.072]$} & 0.267 & 0.222 \\
A2 & {$[0.042,0.072]$} & 0.133 & {$[0.111,0.222]$} \\
A3 & 0.253 & 0.133 & {$[0.056,0.111]$} \\
A4 & {$[0.032,0.063]$} & {$[0.067,0.167]$} & 0.222 \\
A5 & {$[0.042,0.063]$} & 0.133 & {$[0.111,0.222]$} \\
A6 & 0.507 & {$[0.167,0.267]$} & 0.111 \\
\hline \hline & & Local weight & \\
Alternative & C4 & C5 & C6 \\
\hline A1 & 0.308 & {$[0.071,0.143]$} & 0.118 \\
A2 & 0.154 & {$[0.143,0.286]$} & 0.235 \\
A3 & 0.154 & 0.286 & {$[0.235,0.275]$} \\
A4 & {$[0.077,0.154]$} & 0.143 & {$[0.059,0.118]$} \\
A5 & {$[0.077,0.154]$} & {$[0.071,0.143]$} & {$[0.137,0.235]$} \\
A6 & 0.154 & 0.143 & 0.118 \\
\hline
\end{tabular}

In Table 3 and Fig.2, the interval global weights obtained by (6) and (7) are shown. From the widths of global weights, uncertainties of A3 and A6 are smaller than those of A2,A4 and A5. The widths of interval global weights are denoted as index of inconsistency in the given information. The interval global weights are interval probabilities since they satisfy the conditions in (3). They are normalized so that the intervals are not too large or too small and the sum of crisp weights within the intervals is one. Intervals without redundancy are useful and helpful information for a final decision.

Fig.3 illustrates the partial order relation of alternatives based on the obtained interval global weights. It should be noted that interval order relation is defined in [?], where $A=[\underline{a}, \bar{a}] \leq B=[\underline{b}, \bar{b}]$ holds if and only if $\underline{a} \leq \underline{b}$ and $\bar{a} \leq \bar{b}$. $\mathrm{A} 1$ is superior and A4 and A5 are not superior to the other alternatives. The conventional crisp global weights lead the linear order relation, however, the decision maker's evaluations are more likely partial order because of uncertain judgements. The interval global weights are suitable to represent the uncertainty in the decision maker's judgements, because they lead the partial order 
Table 3

Interval global weights of alternatives

\begin{tabular}{ccc}
\hline Alternative & Global weight & Width \\
\hline A1 & {$[0.213,0.233]$} & 0.020 \\
A2 & {$[0.141,0.187]$} & 0.046 \\
A3 & {$[0.191,0.197]$} & 0.007 \\
A4 & {$[0.096,0.150]$} & 0.054 \\
A5 & {$[0.083,0.156]$} & 0.073 \\
A6 & {$[0.169,0.179]$} & 0.010 \\
\hline
\end{tabular}

relation of alternatives.

In Table 4, the selected crisp referenced priority weights of $\mathrm{C} 1, \mathrm{C} 2, \mathrm{C} 3$ and $\mathrm{C} 6$ for the upper bound of interval global weights of A2 and A6 by (6) are shown. As for A2, its local weights under C3 and C6 are large among all alternatives from Table 2. The crisp referenced priority weights of the two criteria are equal to the upper bound of intervals in order to maximize the global weight. As for A6, on the other hand, local weights under C1 and C2 are large among all alternatives, their crisp referenced priority weights are the same as the upper bound of intervals. The referenced priority weights are determined by reflecting each alternative's local weights.

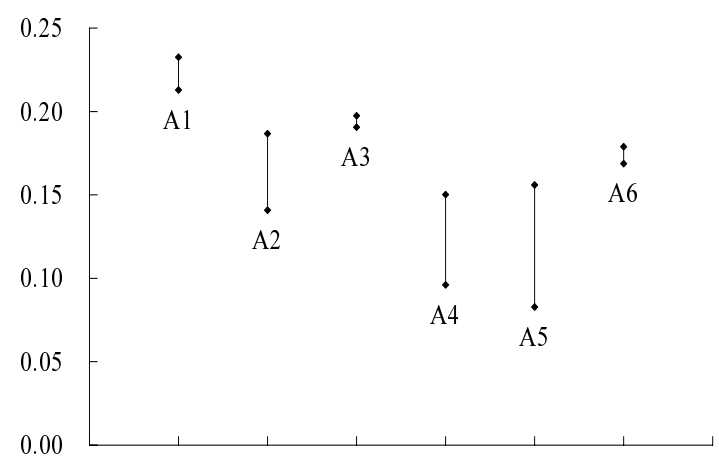

Fig. 2. Interval global weights

Table 4

Selected crisp referenced priority weights for upper bounds of interval global weights of alternatives A2 and A6 by (6)

\begin{tabular}{ccccc}
\hline & $\mathrm{C} 1$ & $\mathrm{C} 2$ & $\mathrm{C} 3$ & $\mathrm{C} 6$ \\
\hline $\mathrm{A} 2$ & 0.008 & 0.090 & $0.072^{*}$ & $0.072^{*}$ \\
$\mathrm{~A} 6$ & $0.056^{*}$ & $0.101^{*}$ & 0.042 & 0.043 \\
\hline
\end{tabular}

* illustrates that it equals to the upper bound of interval referenced priority weight. 


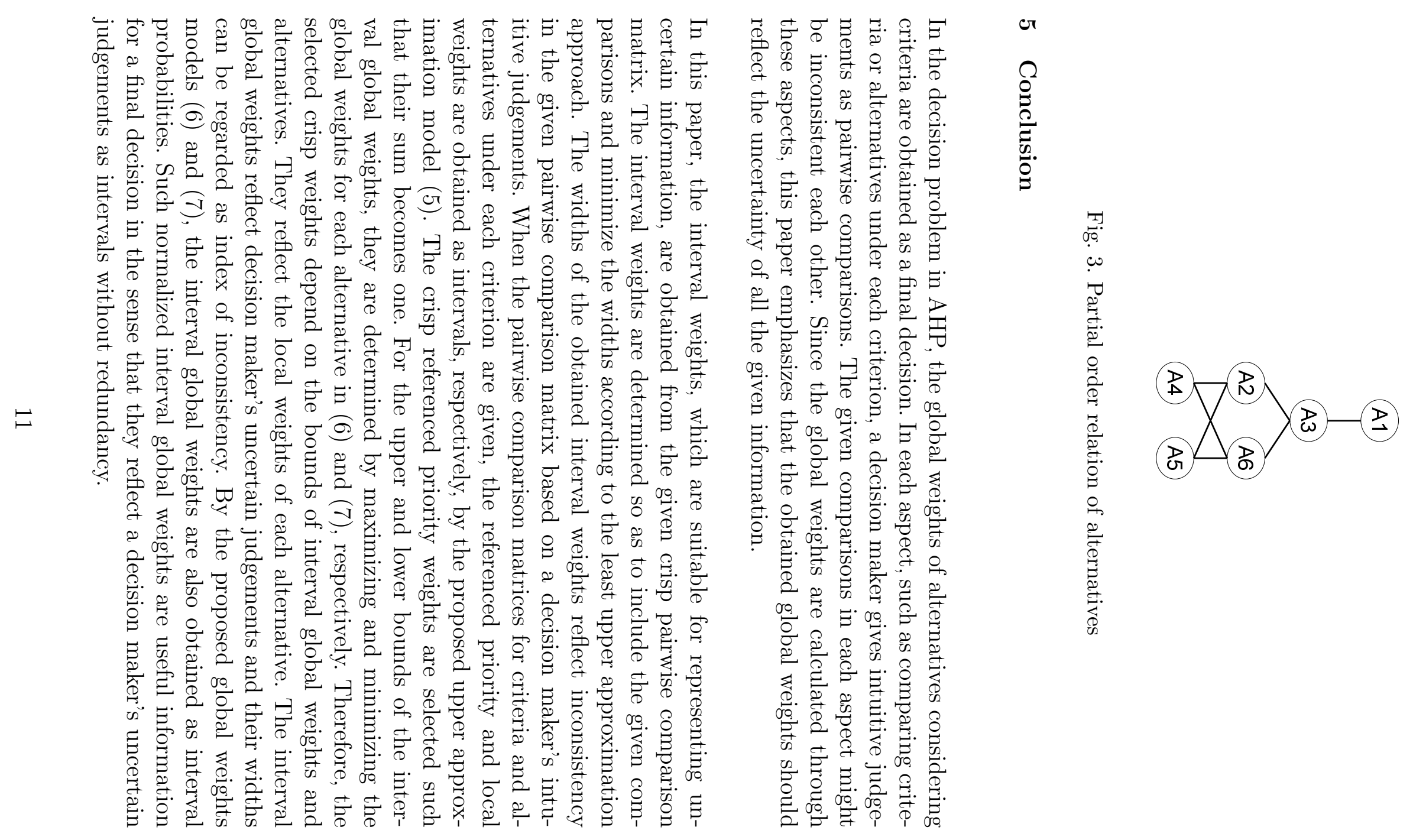




\section{Appendix}

The interval global weight of alternative $\mathrm{A}_{i}$ is obtained as $W_{i}=\left[\sum_{k} p_{k *}^{i} \underline{w}_{k i}, \sum_{k} p_{k}^{i *} \bar{w}_{k i}\right]$ where $p_{k *}^{i}$ and $p_{k}^{i *}(k=1, \ldots, m)$ are the optimal solutions of (6) and (7) for each alternative, respectively. The first condition of interval probabilities (3) is verified as follows.

$$
\begin{aligned}
& \sum_{i \neq j} \sum_{k} p_{k}^{i *} \bar{w}_{k i}+\sum_{k} p_{k *}^{j} \underline{w}_{k j} \\
& \geq \sum_{i \neq j} \sum_{k} p_{k *}^{j} \bar{w}_{k i}+\sum_{k} p_{k *}^{j} \underline{w}_{k j}(\mathrm{i}) \\
& =\sum_{k} p_{k *}^{j}\left(\sum_{i \neq j} \bar{w}_{k i}+\underline{w}_{k j}\right)(\mathrm{ii}) \\
& \geq \sum_{k} p_{k *}^{j}(\mathrm{iii}) \\
& =1
\end{aligned}
$$

(i) The referenced priority weights $p_{k *}^{j}(k=1, \ldots, m)$ are the optimal solutions of (7) for alternative $A_{j}$. They can be the possible solutions of (6) for alternative $\mathrm{A}_{i}$, since the constraint conditions of (6) and (7) are the same. It follows that $\sum_{k} p_{k}^{i *} \bar{w}_{k i} \geq \sum_{k} p_{k *}^{j} \bar{w}_{k i}$.

(ii)The interval local weights $W_{k i}=\left[\underline{w}_{k i}, \bar{w}_{k i}\right](k=1, \ldots, m)$ for each criterion $\mathrm{C}_{k}$ are interval probabilities so that it holds $\sum_{i \neq j} \bar{w}_{k i}+\underline{w}_{k j} \geq 1$ from the condition of interval probabilities (3).

(iii) The sum of the referenced priority weights $p_{k *}^{j}(k=1, \ldots, m)$ are normalized to be one in the constraint condition of (6).

The second condition in (3) is verified in the similar way as follows.

$$
\begin{aligned}
& \sum_{i \neq j} \sum_{k} p_{k *}^{i} \underline{w}_{k i}+\sum_{k} p_{k}^{j *} \bar{w}_{k j} \\
& \leq \sum_{i \neq j} \sum_{k} p_{k}^{j *} \underline{w}_{k i}+\sum_{k} p_{k}^{j *} \bar{w}_{k j} \\
& =\sum_{k} p_{k}^{j *}\left(\sum_{i \neq j} \underline{w}_{k i}+\bar{w}_{k j}\right) \\
& \leq \sum_{k} p_{k}^{j *}=1
\end{aligned}
$$

Then, the interval global weights $W_{i}(i=1, \ldots, n)$ satisfy both of the two conditions in (3). Thus, they are interval probabilities. (Q.E.D.) 


\section{References}

[1] A.Arbel (1989) "Approximation Articulation of Preference and Priority Deviation," European Journal of Operational Research, 43:317-326.

[2] D.Dubois and H.Prade (1980) "Systems of Linear Fuzzy Constraints," Fuzzy Sets and Systems, 3:37-48.

[3] S.Ohnishi, D.Dubois, H.Prade and T.Yamanoi (2006) "A Study on the Analytic Hierarchy Process," Proc. of the 11th International Conference on Information Processing and Management of Uncertainty in Knowledge-Based Systems (IPMU'06), Paris, France.

[4] T.L.Saaty (1980) The Analytic Hierarchy Process, McGraw-Hill, NY.

[5] T.L.Saaty and L.G.Vergas (1987) "Uncertainty and Rank Order in the Analytic Hierarchy Process", European Journal of Operational Research, 32:107-117.

[6] A.A.Solo and R.P.Hamalainen (1995) "Preference Performing through Approximate Ratio Comparisons," European Journal of Operational Research, $82: 458-475$.

[7] K.Sugihara and H.Tanaka (2001) "Interval Evaluations in the Analytic Hierarchy Process by Possibility Analysis," International Journal of Computational Intelligence, 17,3:567-579.

[8] H.Tanaka and P.Guo (1999) Possibilistic Data Analysis for Operation Research, Physica-Verlag, A Springer Verlag Company.

[9] H.Tanaka, K.Sugihara and Y.Maedab (2004) "Non-additive Measures by Interval Probability Functions," Information Sciences, 164:209-227. 